\title{
WAVES AND OSCILLATIONS IN MAGNETIC FLUXTUBES
}

\author{
M.P. RYUTOVA \\ Institute of Nuclear Physics \\ 630090 Novosibirsk, 90 \\ USSR
}

\begin{abstract}
According to observational data solar magnetic fields have a pronounced filamentary structure. Theoretical investigations of plasmas containing structured magnetic fields, including the study of the properties of these structures and their interactions with associated gas flows, are of great importance for our understanding of the basic processes in the solar atmosphere, whose structure and dynamics are dominated by magnetic fields. In the present review theoretical models of thin magnetic fluxtubes and their behaviour in the ambient plasma are discussed.
\end{abstract}

\section{INTRODUCTION}

A situation when the magnetic field is concentrated into randomly distributed bundles of field lines is often met in laboratory and space plasmas. It is well known that all the solar magnetic fields, from the convection zone to the heliosphere, have a pronounced filamentary structure (cf. Stenflo, 1989 [1] and References therein). In the photosphere the magnetic field is concentrated in almost vertical, thin (about $300 \mathrm{~km}$ ) fluxtubes, usually widely separated from each other, with field strengths of the order of 1-2 kG. In sunspots intense (3-4 kG) fluxtubes are assumed to be tightly packed. The isolated fluxtubes are as a rule localized at the supergranular cell boundaries, extending from the subsurface regions through the photosphere and chromosphere, where they form a great variety of magnetic structures. These structures generally have longitudinal dimensions much larger than the transverse ones, and are exposed to the action of a constantly "booming" atmosphere, which results in the generation of different kinds of waves and oscillations. For a better insight into the active processes in the solar atmosphere, such as field concentration, the processes of energy transfer from the lower to the upper layers of the atmosphere, the processes of energy storage and release, preflare and flare processes, etc., the properties of both isolated tubes and ensembles of them should be analysed. Besides its significance for astrophysical objects, such a study is also of interest from the point of view of general physics, due to the wealth of wave processes in such structures. 
In general, the studies of the problem show a rapid progress. There should be mentioned the essential contributions by P.R.Wilson, L.E.Cram, E.Parker, W.Unno, E.R.Priest, N.Weiss, J.Hollweg, H.Spruit, B.Roberts, M.Schüssler and others (see [2] and References therein). But main problems raised by observational evidence are not yet understood, though numerous theories that address these problems have been proposed. The physics of magnetic fluxtubes remains today of great interest. In the present paper I will give a survey of some works which are devoted to the studies of oscillations of magnetic fluxtubes. I will not try to apply these results to explain any particular observational data but there is a hope that the theoretical results which will be presented can give at least some reasonable frame for the analysis of particular situations at the Sun.

As it was mentioned above, the permanently booming atmosphere, in particular, motions in convective zone excite several types of oscillations of fluxtubes. We shall consider long-wave oscillations whose wavelength $\lambda=1 / \mathrm{k}$ is much larger than the radius of magnetic fluxtube $\mathrm{R}: \mathrm{kR} \ll<$. Just these oscillations are most readily excited by large-scale plasma motions and have a relatively low damping rate. Among these oscillations the most important modes are the two shown in Fig.1: a) the bending (kink) oscillations which are actually the dipole mode corresponding to the azimuthal wavenumber $\mathrm{m}= \pm 1$, and $\mathrm{b}$ ) axisymmetric sausage mode $(m=0)$.

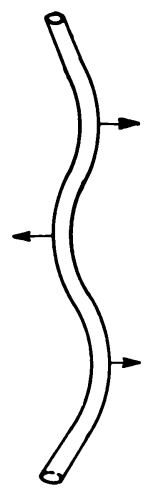

BENDING, $m=1$

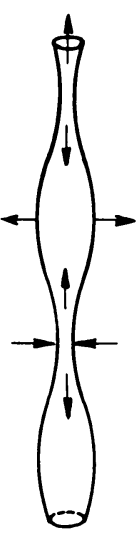

SAL'SAGE, $m=0$
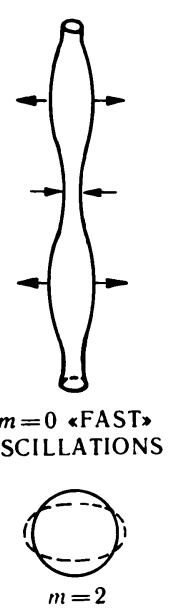

Fig. 1

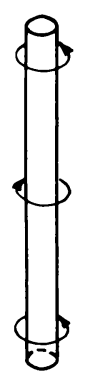

$m=0$ TORQUE OSCILLATIONS<smiles>C1C2CC3CC1CC(C2)C3</smiles><smiles></smiles>

Fig. 2

For both types of oscillations the frequency scales linearly with wavenumber.

The bending oscillations are some analogue of Alfvén waves. As the external plasma participates in the motion in the vicinity of a tube via the "added mass" effect its density enters their dispersion relation (Ryutov \& Ryutova, 1976): 


$$
c_{b}=\frac{\omega}{k_{z}}=\frac{a}{\sqrt{1+\rho_{e} / \rho_{i}}} \text {. }
$$

Here subscript " $i$ " refers to the tube interior, while "e" - to the external plasma; $\rho$ is the plasma density, $a=B / \sqrt{4 \pi \rho_{i}}$ is the Alfvén velocity.

The sausage mode is specific quasi-longitudinal oscillation of fluxtube in which a compression (expansion) of a plasma inside the tube is compensated by the decrease (increase) in the longitudinal magnetic field due to a corresponding change in the cross section of fluxtube, so that the sum of gas-kinetic and magnetic pressures is a1most not perturbed. Thus the plasma parameters outside the tube have little influence on their dispersion relation (giving the corrections of the order of $(k R)^{2}$ ) (Defouw, 1976):

$$
c_{T}=\frac{w}{k_{z}}=\frac{a s_{i}}{\sqrt{a^{2}+s_{i}^{2}}} .
$$

Here $s=\gamma P / \rho \quad$ is a sound speed $(\gamma$ is a specific heat ratio). Some less important modes are shown in Fig. 2. There are high frequency fast oscillations which are analogue of fast magnetosonic waves. As their frequency is extremely high (of the order of $a / R$ ) they can hardly be excited and experience fast radiative damping. The $m=0$ torsional oscillations are just Alfvén wave, but their amplitude is very small (of the order of $\mathrm{V}_{\text {conv. }} \mathrm{R} / \mathrm{L}$, where $\mathrm{L}$ is convective cell size and $\mathrm{V}_{\text {conv. }}$ is characteristic velocity in convective zone) and also are of less interest. The oscillations with the higher azimuthal mode numbers $m=2 ; 3, \ldots$ are very weakly coupled with the large scale motions of medium since the matrix elements which determine this coupling contain a sma11 parameter (kR) $|\mathrm{m}|$.

It should be noted that for theoretical investigation of the properties of fluxtubes and their ensembles (including the model of a spot as a cluster of intense fluxtubes) the concept of a thin fluxtube has proved to be very fruitful and justified. All mentioned above relates to the thin fluxtube model. Even in this concept the physics of plasma containing fluxtubes is very rich.

There exist specific damping mechanisms consisting in the radiation of secondary acoustic waves into external plasma by oscillating fluxtube (Ryutov and Ryutova, 1976), and in resonance excitation of Alfven waves with continuous spectrum in the region where the phase velocity of oscillations is close to local Alfvën velocity (Ryutova, 1977, Ionson, 1978). These effects contribute to the heating of upper layers of atmosphere. The gravity leads to the changing of the character of waves, which become dispersive. Another effect is that in the simple case of isothermal atmosphere amplitude of oscillations increases exponentially with height which leads to the development of strong 
nonlinear effects in tube environment (Spruit, 1981). In stratified atmosphere there appears the frequency cutoff which leads to the existence of propagating (above cutoff frequency) and evanescent (below cutoff frequency) waves (Defouw,1976; Roberts and Webb, 1978,1979). An evanescent wave can be regarded as the quasistatic response of a tube to the slow changes in the region of wave excitation. If the wave is generated impulsively at the base of a tube it propagates with height in the form of a wave front with velocity of corresponding wave mode. Behind the wave front is trailed a wake oscillating at the cutoff frequency (Rae and Roberts, 1982). For the compressive case the formation of shocks both from the wave front and from the oscillating wake takes place (Hollweg, 1982).

When fluxtube parameters have smooth radial dependence there appear very peculiar evolution of the radial mode structure of tube waves: it becomes more and more spiky at higher altitudes and respectively the dissipative processes become important, which results in a faster damping of the wave. The longitudinal dependence of the energy flux becomes nonexponential. Statistical analysis of the case when tube experiences random oscillations caused by photospheric convective motions shows that even "white noise" of convective zone can result in a temporal brightening of the tube region at definite height (Ryutova, 1989).

Very interesting physics is brought about by taking into account the presence of shear flow along the fluxtubes. The plasma flows with different velocities inside and outside fluxtube gives rise to the qualitatively new effects: the appearance of negative energy waves, reversal of the sign of radiative damping, the development of explosive instability at nonlinear stage, and the development of linear hydrodynamic instability similar to instability of tangential discontinuity (Ryutova, 1988).

The picture of nonlinear effects in structured magnetic fields even at the stage which we have today (with a great work yet to be done) is very rich. Roberts and Mangeney (1982) have shown that when gravity is ignored in a slab geometry the Benjamin-Ono type solitons can appear. After this paper, solitary waves in a slab and cylindrical tube are extensively studied. Roberts (1985) showed that slow surface waves are governed by nonlinear integrodifferential equation which possesses the soliton solution. Edwin and Roberts studied Benjamin-Ono-Burgers type equation and concluded that the estimation of the damping rate of corresponding solitary wave enables these waves to propagate from lower layers to upper chromosphere unharmed. Molotovshchikov and Ruderman (1987) obtained the equations describing the long nonlinear sausage waves (both slow and body) in fluxtube in magnetic environment. Note that under the conditions when negative energy waves are excited in the system the solitary waves with explosively growing amplitude can appear. Another class of nonlinear effects is connected with the backward effect of longwave oscillations on plasma which consists in the generation of secondary plasma flows and electric currents. These phenomena lead to macroscopic effects which can play an essential role in the dynamics of structured magnetic fields and which, in principle, can be observed. The effect of secondary plasma flows (Ryutova, 1986) is si- 
milar to the effect of "acoustic" or "quartz wind" in usual hydrodynamics but its picture in MHD is more complicated. At the propagation of longwave oscillations along the fluxtube there arise stationary vortex flows in the plane perpendicular to the magnetic field and upward mass flows along the field. The main effect of the "magnetosonic wind" is that the fluxtube is vanishing "diffusively" or is splitting into thinner independent tubes. If the absorption of oscillation energy is determined mostly by one of the plasma components then besides the generation of "magnetosonic wind" it is accompanied by the excitation of currents. The effects mentioned above are connected with the oscillations of fluxtubes. It is worth mentioning that there exists another mechanism of current drive and, respectively, of a generation of magnetic fields which is not necessarilyconnected with fluxtube oscillations. Generally speaking the absorption of the momentum of usual acoustic waves generated in solar atmosphere results in a transfer of the momentum to plasma electrons and ions. It is shown that in a collisional case of solar chromosphere where damping of acoustic waves is caused by the nonlinear effects (formation of weak shocks) quite strong currents and magnetic fields are generated (Ryutov \& Ryutova, 1989).

The problem connected with the properties of ensemble of magnetic fluxtubes is explored much less than the properties of separate fluxtube. Ryutov and Ryutova (1976) studied the propagation of sound waves in a plasma containing an ensemble of randomly distributed magnetic fluxtubes and found that even in the absence of any dissipative effects (viscosity, thermal conductivity, Ohmic losses) sound waves are absorbed due to the effect similar to Landau damping and consisting in the resonance excitation of fluxtube oscillations. In a much longer time than the time during which the energy of outer motions is transferred into the energy of fluxtube oscillations magnetic tubes release their energy in the form of secondary sound waves into the upper layers of atmosphere. The contribution of noncollinearity of fluxtubes into the resonant absorption and resonant scattering of sound waves is found. Quite recently Bogdan (1989) studied the resonance scattering of sound waves by fluxtubes paying the most attention to the interaction of solar p-mode with fluxtubes and indicating the importance of this effect as a diagnostic probe of the structure of solar magnetic flux concentrations (see also Bogdan \& Zweibe1, 1985, 1987; Bogdan \& Cataneo, 1989). Ryutova \& Persson (1984) studied dispersion properties of a plasma containing small scale random inhomogeneities when density and magnetic field change by the order of unity at a length small compared to the wavelength (the model of a spot as a cluster of intense fluxtubes). Unlike the case of homogeneous plasma the wave propagation in such system is accompanied by vortex motion of plasma having the same scale as that of the nonhomogeneities. The main effect which takes place in such a clusters of fluxtubes is the enhanced dissipation of MHD waves caused by large local gradients of temperature, velocity,etc.

of course, the present review is far from complete and in addition to References presented here I end the Introduction with some more of them. Namely, the papers of N.O.Weiss (1981), Hasan \& Schüssler (1985), Ferriz-Mas (1988), Hollweg (1987), Hollweg et al. (1989), Seehafer 
(1988), Henoux \& Somov (1987), and earlier reviews by Spruit (1981), Thomas (1985) and Priest (1988).

\section{LINEAR EFFECTS}

\subsection{Some basic properties of fluxtube oscillations}

The study of fundamental modes of fluxtube oscillations is based on the linearized MHD-equations complemented by the equilibrium condition of fluxtube in unperturbed state

$$
P_{i}(r)+\frac{B^{2}(r)}{8 \pi}=P_{e} \text {. }
$$

Here $P_{i}$ and $P_{e}$ are gas-kinetic pressures inside and outside the tube and $B$ is the magnetic field inside the tube. The condition (3) is written for cylindrical fluxtube embedded in a field free medium. For the perturbations proportional to $\exp (-i \omega t+i k z+i m \varphi)$ (we use the cylindrical coordinates with z-axis coinciding with tube axis) in the absence of gravity the phase velocities of two fundamental modes, kink $(m=1)$ and sausage $(m=0)$, have a form (1) and (2), respectively. When gravity is included there appears a buoyancy force which changes the character of tube oscillation.

For isothermal case neglecting the compressibility of medium the equation for kink oscillations can be written as (Spruit, 1981a,b):

$$
\frac{\partial^{2} \xi}{\partial t^{2}}=c_{b}{ }^{2} \frac{\partial^{2} \xi}{\partial z^{2}}+g\left(\frac{\rho_{i}-\rho_{e}}{\rho_{i}+\rho_{e}}\right) \frac{\partial \xi}{\partial z} ;
$$

$\xi$ is the transverse displacement of the tube (which is much larger for these oscillations than the vertical displacement of the plasma inside the tube). The solution of Eq. (4) gives for the amplitude

$$
\xi \sim e^{-i \omega t}+i k z+\frac{z}{4 H}
$$

and for the frequency

$$
\omega^{2}=c_{b} k^{2}=\frac{c_{b}^{2}}{4 H}
$$

where $H$ is a pressure scale height and $\omega_{c}^{b}=\frac{c_{b}}{4 H}$ is the cutoff frequency.

The same holds for the sausage oscillations. The corresponding equations written for the vertical component of velocity which for these oscillations in contrast with the kink mode is much larger than the transverse one, have a form (Defouw): 


$$
\begin{aligned}
& \rho \frac{\partial v_{z}}{\partial t}+\rho v_{z} \frac{\partial v_{z}}{\partial z} \simeq-\frac{\partial P}{\partial z}+\rho g \\
& \frac{\partial}{\partial t}\left(\frac{\rho}{B}\right)+\frac{\partial}{\partial z}\left(\frac{\rho u}{B}\right)=0 .
\end{aligned}
$$

These equations together with the equilibrium condition of a thin fluxtube (3) give for the isothermal atmosphere the following dispersion relation:

$$
\omega^{2}=c_{T}{ }^{2} k^{2}+\left(\omega_{c}^{T}\right)^{2}
$$

with the cutoff frequency

$$
\omega_{c}^{T}=\frac{c_{T}}{4 H}\left(9-\frac{8}{\gamma}+\frac{16(\gamma-1)}{\gamma^{2}} \frac{s^{2}}{a^{2}}\right)^{\frac{1}{2}} .
$$

As to solution for the velocity amplitude it has the same form as (5). (Appropriate studies for the arbitrary temperature profile $T(z)$ see Roberts and Webb (1978); for the case of variable specific heat ratio and the mean molecular weight see Spruit and Zweibel (1979).

Thus, the dispersive nature of both modes in isothermal atmosphere is analogous to that of an acoustic wave propagating vertically upward. The waves with the frequencies $\omega<\omega_{c}$ are evanescent. For propagating waves the frequency cutoff works as a filter which lets through the thickness of atmosphere only those waves among the great variety of waves excited in convective zone whose frequency is larger than $\omega_{c}$. Since for bending oscillations the cutoff is quite low these waves are expected to reach chromosphere with growing amplitudes (in accordance with Eq. (5)) (Spruit, 1981a). Rae and Roberts have shown that if disturbances of fluxtube are impulsively generated at its base, then the wave propagation occurs and the existence of cutoff manifests itself in the formation of an oscillating wake. The wake is oscillating with the tube frequency.

The above conclusions are valid for isothermal atmosphere. In a non-isothermal atmosphere fluxtube oscillations may become unstable. For a thin tube embedded in a convective zone the instability is driven by the buoyancy force in much the same way as in ordinary convection (Webb \& Roberts, 1978; Spruit, 1979; Spruit \& Zweibe1, 1979). The stability criterion (actually Schwarzschild's criterion) is modified by the presence of magnetic field. Namely, the magnetic field exerts the stabilizing influence and constrains the unstable motion to a flow along the tube. If the instability sets in as a downdraft it leads to a new equilibrium state of higher field strength, but a lower total energy. The magnetic energy is increased at the expense of the gravitational energy of plasma within the tube.

One more example of changing the character of tube oscillations is that when there is a sharp discontinuity in the vertical temperature distribution - the situation typical for chromosphere and the transition 
zone. The pressure balance condition means that in this region a steep drop of plasma density inside the tube occurs providing the condition for the wave reflection and for the formation of resonance structure in the open fluxtube. This means that in the region of the temperature jump one can expect quite a large oscillation level. Since the lower point of fluxtube experiences random oscillations caused by the convective motions, besides the study of boundary value problem one needs to analyse the behaviour of the spectral density of tube oscillations. The appropriate analysis shows that with a considerable probability the amplitude of tube oscillations can be 3 or 4 times larger than its average value which by itself, in the region of temperature jump becomes much larger than the amplitude of motions in convective zone (Ryutova, 1989). In the observational data such events can manifest themselves as a temporal brightening of tube region. The same holds for another mechanism of the formation of 1-D resonator in open fluxtube which is connected with the rapid radial broadening of the tube at some altitude caused by the loss of radial equilibrium. As is well known, such phenomenon occurs when the plasma temperature outside the tube is less than that inside. The rapid broadening gives rise to a growing tube inertia which results in the "pinning" of the tube at the altitude of the loss of equilibrium.

Up to now, we considered the fluxtubes homogeneous in their cross section. In the next section we will see that the radial dependence of tube parameters leads to a number of new effects. In the same section we consider the influence of compressibility of medium.

\subsection{Anomalous and radiative damping of fluxtube oscillations}

The allowance for a smooth profile of plasma parameters over the tube radius brings about the specific (and strong) damping of oscillations which is caused by the resonance between the phase velocity of oscillations and the local Alfvén velocity. Mathematical form of corresponding equation is of the type of Rayleigh equation in usual hydrodynamics with the coefficient of the higher derivative approaching zero in singular point. Beginning with the paper of Timofeev (1970) such type of equation and corresponding effects, in particular Alfvén resonance, in laboratory plasma is studied in detail. The effect of Alfvén resonance appeared to be very important in physics of coronal loops and heating processes (Ionson,1978; Hollweg,1979; Heyvaerts and Priest, 1983; see also Sudan and Similon, 1988). Just for magnetic fluxtube oscillations this effect was studied by Ryutova (1977) who considered longwave bending (kink) oscillations in an incompressible limit. In this case the MHD-equations come to a single equation for current function $\psi$ :

$$
\frac{\partial}{\partial r}\left(\rho-\frac{k^{2} B^{2}}{4 \pi \omega^{2}}\right) r \frac{\partial \psi}{\partial r}-\left(\rho-\frac{k^{2} B^{2}}{4 \pi \omega^{2}}\right) \frac{\psi}{r}=0
$$

(current function relates to the velocity components by $v_{r}=\frac{1}{r} \frac{\partial \psi}{\partial \varphi}$ and 
$\left.v_{\varphi}=\frac{\partial \psi}{\partial r}\right)$. For the arbitrary radial profiles of density and magnetic field the quantitative evaluation of the damping rate is not quite simple. The eigenvalue problem can evidently be solved for the model of a tube which is homogeneous everywhere except the narrow (but finite) boundary layer of the width $\varepsilon R$, where the Alfvén and sound speeds are linear functions of radius ( $\varepsilon$ is a small parameter. For this model the damping rate is proportional to $\varepsilon$ (qualitatively, if $\varepsilon$ is of the order of $\mathrm{R}$, the damping rate becomes comparable with the frequency):

$$
\nu_{\text {res }}=\frac{\pi \varepsilon}{4} \frac{\rho_{i}}{\rho_{i}+\rho_{e}}
$$

Note that the anomalous damping, by itself, does not transform the energy of oscillations to the plasma heating. It just results in the concentration of the initially smooth eigenfunction near the resonant point, or, in other words, to a conversion of oscillation energy to the Alfvén continuum. But then, of course, the usual dissipation mechanisms, like viscosity and others, turn on, and this strongly oscillating distribution damps out.

Returning to the case of a really smooth radial dependence of fluxtube's parameters note that the weakly damped wave which could be described in terms of a smoothly varying radial eigenfunction does not exist any more. As in a real situation the radial distributions in most cases should be smooth, the question arises of whether under such conditions the bending oscillations of fluxtube can still be an agent responsible for the energy transfer from the underlying surface to upper chromosphere. To answer this question one should study a boundary value problem for the tube excited in its footpoint. The appropriate analysis in a most general case of a profile without any small parameter shows (Ryutova, 1989) that at higher altitudes the radial structure of the perturbation which was smooth near the excitation point, becomes more and more spiky at higher altitudes. The characteristic radial scale length $\Delta \mathrm{r}$ diminishes inversely proportional to $\mathrm{z}$ :

$$
\Delta \mathrm{r} \sim \mathrm{Ra} / \mathrm{\omega z} \text {. }
$$

Respectively, due to the presence of these small scale structures the dissipative processes (viscosity, thermal conductivity, etc.) become more and more important at larger $z$. The longitudinal dependence of the energy flux of the oscillations becomes nonexponential. The corresponding estimates give

$$
Q \sim \rho \nu \frac{\omega^{2} \xi^{2}}{(\Delta r)^{2}},
$$

where $Q$ is a volume density of a power released by viscous dissipation ( $\nu$ is a kinematic viscosity coefficient). As $\Delta r$ diminishes with height, 
$Q$ is growing with $z$. At some altitude $z^{*}$ reaches maximum, and then rapidly decreases - just because the amplitude of oscillation decreases. Thus, the heating power has a very characteristic shape with a pronounced maximum at some altitude $z^{*}$. For comparison the dotted line in Fig. 4 shows $Q$ for usual exponentially damping wave. The estimate of the altitude $z$ * is

$$
z^{*} \sim \frac{\mathrm{a}}{\omega}\left(\frac{\mathrm{R}^{2} \omega}{v}\right)^{1 / 3}
$$

Thus, one can conclude that even in the case of a smooth radial profile of plasma density and magnetic field the bending waves can transfer energy from underlying surface to upper chromosphere.

Let us proceed now to another important feature of fluxtube oscillations connected with the compressibility of medium. Ryutov and Ryutova (1976) have shown that in compressible medium oscillating fluxtube can be a source of secondary acoustic waves. The frequency of the radiated acoustic wave is obviously equal to that of the fluxtube oscillations. The same holds for the longitudinal wavenumber $k$. The radial wavenumber of acoustic wave is determined from the dispersion relation and is equal to:

$$
k_{r}=\frac{1}{s_{e}}\left(\omega^{2}-k^{2} s_{e}\right)^{\frac{1}{2}}
$$

Here $s_{e}$ is the sound speed in the external plasma.

The radiation occurs if $k_{r}$ is real, that is if the phase velocity $\omega / k$ of flux tube oscillations is larger that $s_{e}$. In the opposite case the acoustic waves are evanescent, and their presence does cause only a small change of phase velocity of tube oscillations. So, for the case when $k_{r}$ is real radiative damping rate of an arbitrary mode (except the axisymmetrical one) is as follows (Ryutov and Ryutova,1976):

$$
\frac{\alpha_{\mathrm{rad}}^{(\mathrm{m})}}{\omega}=\frac{\pi}{|\mathrm{m}| !(|\mathrm{m}|-1) !(1+n)}\left(\frac{\mathrm{Rk}}{2}\right)^{2|\mathrm{~m}|}\left[\frac{2}{\gamma(1+n)}-1\right]^{|\mathrm{m}|} .
$$

Respectively for bending oscillations we have

$$
\frac{\alpha_{\mathrm{rad}}^{(1)}}{\omega}=\frac{\pi}{1+\rho_{i} / \rho_{e}}\left(\frac{\mathrm{Rk}}{2}\right)^{2}\left[\frac{2}{\gamma\left(1+\rho_{i} / \rho_{e}\right)}-1\right] .
$$

For sausage oscillations (Ryutova, 1981):

$$
\frac{\alpha_{\mathrm{rad}}^{(0)}}{\omega}=\frac{\pi}{2}\left(\frac{\mathrm{Rk}}{2}\right)^{2} \frac{\mathrm{s}_{i}{ }^{6}}{\mathrm{~s}^{2}\left(\mathrm{a}^{2}+\mathrm{s}_{i}{ }^{2}\right)^{2}} .
$$


The damping rate contains in all the cases a small parameter $(k R)^{2}$ and thus is relatively small. This smallness is a reflection of the fact that the frequency of tube oscillations is small as compared to the eigenfrequency of its radial acoustic oscillations. For short wavelengths the damping rate becomes large, of the order of frequency. This is another reason why just the longwave oscillations are of the most interest.

So, approaching the real conditions of solar atmosphere we have taken into account the gravity effect, the radial inhomogeneity of fluxtube and the compressibility of medium. The next step is taking into account the presense of longitudinal mass flows along the magnetic structures.

\subsection{Effect of sheared flows}

As it was mentioned in Introduction, the presence of longitudinal flow of a plasma in the vicinity of fluxtube gives rise to qualitatively new effects (Ryutova, 1988). First of all, when the velocity of shear flow exceeds a certain threshold value there arise negative energy waves in the system which can become unstable due to various energy absorption processes including nondissipative mechanisms of damping. In the case of fluxtubes just because of their specific feature described above the instability can occur due to the radiation of secondary sound waves and due to anomalous damping in Alfvén resonance region. When the velocity exceeds the second threshold there arises the linear hydrodynamic instability similar to the instability of the tangential discontinuity (TD) in MHD or Kelvin-Helmholz instability in hydrodynamics. Note, that according to observational data the plasma flows usually with different velocities inside and outside the magnetic structures are observed in all the magnetized regions in solar atmosphere.

In the coordinate system where the substance inside the tube is at rest, while the flow velocity outside it equals $u$ and is directed along the z-axis, the linearized MHD-equations together with the equilibrium condition (3) lead to the following set of equations for small oscillations of magnetic fluxtube in the presence of shear flow:

$$
\begin{aligned}
& i \delta \mathfrak{S}=\rho(r) \frac{\Omega^{2}(r)\left(s^{2}+a^{2}\right)-k^{2} s^{2} a^{2}}{n^{2}-k^{2} s^{2}}\left[\frac{1}{r} \frac{\partial}{\partial r} r \frac{v_{r}}{\Omega(r)}+\frac{i m}{r} \frac{v_{\varphi}}{\Omega(r)}\right] \\
& \frac{\partial \delta \Re}{\partial r}=i \rho(r)\left[\Omega^{2}(r)-k^{2} a^{2}\right] \frac{v_{r}}{\Omega(r)} \\
& \frac{i m}{r} \delta S=i \rho(r)\left[\Omega^{2}(r)-k^{2} a^{2}\right] \frac{v_{\varphi}}{\Omega(r)} .
\end{aligned}
$$

Here $\delta \mathfrak{S}=\delta \rho+b_{z} B / 4 \pi$ is the total pressure perturbation (all perturbed quantities are assumed to be proportional $\exp (-i \omega t+i k z+i m \varphi))$, and

$$
\Omega(r)=\omega-k u(r) \text {. }
$$


The set (11) describes all the types of linear oscillations of magnetic fluxtube. (For $u=0$ they were described above). To illustrate the properties of negative energy waves we consider the bending oscillations. In this case the set (11) is reduced to the following equation for the displacement vector (cf. Eq. (4)):

$$
\rho_{i} \frac{\partial^{2} \vec{\xi}}{\partial t^{2}}=-\rho_{e}\left(\frac{\partial}{\partial t}+\frac{\partial}{\partial z}\right)^{2} \xi+\frac{B^{2}}{4 \pi} \frac{\partial^{2} \xi}{\partial z^{2}} .
$$

The dispersion relation following from (12) has a form:

$$
\omega^{2}+\frac{1}{\eta}(\omega-k u)^{2}-k^{2} a^{2}=0
$$

where $\eta=\rho_{i} / \rho_{e}$. From (13) we have

$$
\frac{\omega}{k}=\frac{1}{1+n}\left\{u \pm \sqrt{n\left[a^{2}(1+n)-u^{2}\right]}\right\} .
$$

Before describing the instability of negative energy waves let us examine the region, where

$$
u>a \sqrt{1+n} \text {. }
$$

This is a region of TD-instability which can be referred as a "coarse" one since its growth rate is comparable with the frequency when $u$ exceeds the threshold by a factor of two. This instability must play an essential role in different astrophysical objects where there are high speed streams along the magnetic field. In particular, the excitation of solar fluxtube oscillations is thought to be caused by shaking of tube's footpoint in convective zone. The frequency of these oscillations is very low, which presents the problem in an attempt to explain the energy transfer from photosphere to upper layers. The TD-instability leads to existence of another mechanism of excitation of oscillations magnetically structured media which is independent of the motions in the base of fluxtube and which can work far from convective zone and moreover, in any region with the strong mass flow. So, if in some region a fluxtube is "blown out" by the upward flow the oscillations excited here propagate further upwards. The frequency of these oscillations can be much higher than the inverse time of re-arrangement of granulation picture.

In the region $u>a \sqrt{1+\eta}$ when system is still stable with respect to TD-instability the instability of negative energy waves can occur. For the lower branch (sign "minus") in dispersion relation (14) the energy density has a form

$$
W=\pi R^{2} k^{2} \rho e^{\xi^{2}}\left(n a^{2}-u^{2}\right) \frac{x}{x+u}
$$

where $\mathrm{x}=\sqrt{\eta\left[\mathrm{a}^{2}(1+\eta)-\mathrm{u}^{2}\right]}$. 
It is seen that the wave energy becomes negative at

$$
u>a \sqrt{n}
$$

Therefore, within the interval

$$
a \sqrt{n}<u<a \sqrt{1+n}
$$

there can exist the instability of NEW caused by any of dissipative processes. In other words, dissipative effects lead to loosing the energy of NEW and, hence, to the growth of their amplitude. Note, that the possibility of the existence of negative energy waves in nonequilibrium plasma for the first time was pointed out by Kadomtsev et al. (1964); on the negative energy waves in hydrodynamics see, for example, Ostrovsky et a1. (1986).

It is remarkable, that for magnetic fluxtubes this instability can occur even in the absence of any usual dissipative processes due to their specific features mentioned above. First, the instability takes place due to the damping in the Alfvén resonance layer. Recently, similar results have been obtained by Hollweg et al. (1989), who studied the effects of velocity shear on the resonance absorption of incompressible MHD surface waves and indicated the importance of this effect for the development of turbulence in regions of strong velocity shear. I would like to emphasize that the strong instability takes place not only for axisymmetrical waves but also for bending waves (see below). Second, in the case of compressible medium the instability occurs due to the radiation of secondary sound waves. Note again, that the development of NEW-instability leads to the reversal of the sign of radiative damping and to the growth of the fluxtube oscillations amplitude. This is possible in two cases: when fluxtube oscillation has a negative energy, while a radiated sound wave has a positive one, or when former has a positive energy, while a radiated wave has a positive one. For example, the conditions under which bending oscillations of positive energy radiate negative energy sound waves are as follows:

$$
\mathrm{a}>\mathrm{s}_{\mathrm{e}} / \sqrt{n}, \quad \mathrm{u}>\mathrm{s}_{\mathrm{e}}+\sqrt{\mathrm{a}^{2}-\frac{1}{\eta} \mathrm{s}_{\mathrm{e}}^{2}} .
$$

As to sausage oscillations the outer flow has a weak influence on them; in particular, their energy remains positive in the presence of flow. So that the instability in this case can be caused by the radiation of negative energy sound waves. The necessary condition has the form:

$$
\mathrm{c}_{\mathrm{T}}=\mathrm{u}-\mathrm{s}_{\mathrm{e}} \sqrt{1+\mathrm{k}^{2} / \mathrm{k}}>0
$$

which is possible when fulfilling the requirement

$$
\mathrm{u}>\mathrm{c}_{\mathrm{T}}+\mathrm{s}_{\mathrm{e}} \text {. }
$$

The most important feature of negative energy waves is that their presence leads to enlargement of classes of instabilities and one can 
expect a very vigorous nonlinear activity in a system. A few of them we describe in the next section.

\section{NONLINEAR EFFECTS}

\subsection{Explosive instability}

In the system where the waves with opposite signs of energy are simultaneously present the nonlinear explosive instability can develop. This instability was first considered by Dikasov et al. (1965) and illustrated by the waves with random phases. Later on, it was analysed by Coppi et al. (1969) for a triplet of coherent waves.

As is well known, the main feature of this instability is that the amplitudes of interacting waves achieve infinitely large values for a finite period of time. As an example let us consider an explosive instability for a triplet of waves propagating along the fluxtube.

Let wave 1 be of a sausage type, thus having a positive energy, (as was mentioned above, external flows have a weak influence on this mode), wave 2 - of bending type with negative energy, that is propagating against the flow and having $k_{2}$ negative, and wave 3 - of bending type with positive energy $\left(k_{3}>0\right)$. The bending wave can be either linearly polarized, that is be a mixture of waves with $\mathrm{m}=1$ and $\mathrm{m}=-1$, or circularly polarized in opposite directions.

The matching conditions can be satisfied if shear flow velocity exceeds some critical value equal to

$$
u_{c}^{\exp }=\frac{c_{T}}{1+\eta}+\left[a^{2} \eta-c_{T}^{2} \frac{\eta\left(\eta^{2}+3 \eta+3\right)}{1+\eta^{2}}\right]^{\frac{1}{2}} \text {. }
$$

It is easy to verify that the value (20) is below the limit of hydrodynamic instability and just gets into the interval corresponding to the existence of negative energy waves determined by (18). So, under the condition

$$
a \sqrt{n}<u<u_{c}^{\exp }
$$

one can expect a very vigorous nonlinear activity in system. So, if only one wave (say wave 1 ) is excited in the system at the initial moment of time and the amplitudes of two other waves are determined by thermal noise, then at the initial stage of development of the explosive instability these amplitudes increase exponentially. The characteristic growth rate by the order of magnitude is equal to $k_{1} v_{1} \sim$, where $v_{1}$ is the velocity amplitude of tube's boundary in slow oscillations. In a time of the order of several inverse growth rates when the amplitudes of all three waves become the quantities of the same order, there begins the power growth of the amplitudes of all interacting waves according to the law

$$
v_{\sim} \sim \frac{1}{t-t_{0}}
$$

and the amplitudes of all three waves achieve infinitely large values 
in a time $t_{0}$, which in our case is also of the order of $\left(k_{1} v_{1}\right)^{-1}$. Of course the assertion made above is formal to some extent: higher-order nonlinear processes can limit the growth of amplitudes at a finite level. So, the further development of explosive instability depends on the character of these nonlinear processes as well as the dispersive properties of system. There can be, for example, "real explosion", that is fast $\left(\sim t_{0}\right)$ release of energy stored in a system; the stabilization of instability with appearance of solitary wave; gas of solitons; solitons with explosively growing amplitudes; shocks; turbulence, etc. The detailed analysis of these processes is the future problem. What is well known today is that nonlinear equations describing fluxtube oscillations possess soliton-type solutions which will be shortly presented in the next section.

\subsection{Solitons in magnetic fluxtubes}

$\mathrm{Eq}$. (7) for sausage together with the equilibrium condition (3) form hyperbolic equations which possess the shock solution. The allowance of the compressibility of medium leads to dispersion of the wave. If the nonlinearities responsible for the creation of shocks are counter-balanced by the dispersive effects the solitons can appear. Roberts (1985) has shown that sausage waves in a thin cylindrical fluxtube satisfy the nonlinear integrodifferential equation which is analogous to the equation which was got by Leibovich (1970) for the description of water waves on a vortex and which has a solitary solution. Earlier such an equation for a slab geometry was obtained by Roberts and Mangeney (1982):

$$
\frac{\partial v_{z}}{\partial t}+c_{T} \frac{\partial v_{z}}{\partial z}+\alpha_{1} v_{z} \frac{\partial v_{z}}{\partial z}+\alpha_{2} \frac{1}{\pi} \frac{\partial^{2}}{\partial z^{2}} \int_{-\infty}^{\infty} \frac{v_{z}(s, t)}{s-z} d s=0
$$

where

$$
\alpha_{1}=\frac{\left[(\gamma+1) a^{2}+3 s_{e}^{2}\right] a^{2}}{2\left(s_{e}^{2}+a^{2}\right)^{2}} ; \alpha_{2}=\frac{1}{2} \frac{\rho_{e}}{\rho_{i}}\left(\frac{c_{T}}{a}\right)^{3} x_{0} c_{T} \text {. }
$$

Here $2 \mathrm{x}$ is the width of the magnetic slab.

Eq. (21) is the Benjamin-Ono type and the corresponding solution is

$$
v_{z}(z, t)=\frac{v_{0}}{1+(z-s t)^{2} / 1^{2}}
$$

where the amplitude $\mathrm{v}_{0}$, speed $\mathrm{s}$ and scale of soliton are related by

$$
\mathrm{s}=\mathrm{c}_{\mathrm{T}}+\frac{1}{\mathrm{u}}, \quad 1=4 \alpha_{2} / \mathrm{v}_{0} \alpha_{1}
$$

This solution corresponds to a swelling in the slab which propagates with a speed $s$. The cross section of a slab depends on $v_{z}$ as 


$$
\mathrm{A}=\mathrm{A}_{0}\left(1+\frac{\mathrm{C}_{\mathrm{T}}}{\mathrm{a}} \mathrm{v}_{\mathrm{z}}\right) \text {. }
$$

Nonlinear analysis including dissipative effects lead to equation of the Benjamin-Ono-Burgers type (Edwin and Roberts, 1986). The estimates of the decay rate of corresponding solution show that solitons can propagate through the nonadiabatic atmosphere and to emerge at higher altitudes.

\section{3. "Magnetosonic flows"}

As was described above, in plasma containing structured magnetic fields a great variety of wave processes take place. This leads to very interesting nonlinear effects analogous to the effect of "acoustic flows" or "quartz wind" in usual gasdynamics (see, for example, Nyborg, 1965 and References therein). But in magnetohydrodynamics the picture of these effects is much more rich (Ryutova, 1986). Namely, at the propagation of oscillations along the fluxtubes secondary plasma flows and currents are excited inside the tube as well as outside it. There are two main reasons leading to these effects: the action of ponderomotive force on plasma (this mechanism is not connected with the absorption of oscillations and with any dissipative processes) and absorption of momentum and angular momentum of oscillations propagating along the tubes (nonzero angular momentum can be transferred by the circularly polarized bending oscillations). The absorption of angular momentum causes the rotational mass flow around the fluxtube and the absorption of longitudinal momentum can lead to the upward mass flow. If the absorption is provided mostly with one of the plasma components (the specific of situation depends on the damping mechanism), then it is accompanied by the excitation of currents which can distort the initial magnetic field.

The velocity field of generated secondary flows is described by the following equation

$$
\rho \frac{\partial \overrightarrow{\mathrm{u}}}{\partial \mathrm{t}}+\rho \nu \nabla^{2} \overrightarrow{\mathrm{u}}=-\nabla\left(\mathrm{P}+\frac{\mathrm{B}^{2}}{8 \pi}\right)+\overrightarrow{\mathrm{f}}
$$

where $\nu$ is kinematic viscosity and $\overrightarrow{\mathbf{f}}$ is ponderomotive force acting on the unit volume of plasma. $\overrightarrow{\mathrm{f}}$ has a following form:

$$
\overrightarrow{\mathrm{f}}=-<\tilde{\rho} \frac{\mathrm{d} \tilde{\overrightarrow{\mathrm{v}}}}{\mathrm{dt}}>+\frac{1}{\mathrm{c}}<[\tilde{\tilde{\mathrm{j}}} \overrightarrow{\vec{B}}]>\text {. }
$$

Here tilde marks perturbed quantities defined by linearized MHD-equations corresponding to one of the modes (bending or sausage). One can see from equation (12) that convective motions can arise only if

$$
\operatorname{rot} \overrightarrow{\mathrm{f}} \neq 0 \text {. }
$$

Otherwise, the ponderomotive force leads only to an insignificant re= distribution of plasma density and magnetic field. The magnitude of $\vec{f}$ becomes especially large in Alfvén resonance layer where quite strong 
convective motions can arise.

These secondary ("magnetoacoustic") flows can bring about different evolution regimes of magnetic fluxtubes which depends on the correlation properties of the wave trains propagating in the tube and on the parameters of fluxtube itself. If we consider a case of long coherent train with the duration $T$ much in excess of the time of establishing of viscous flow $\left(\tau_{\nu} \sim \mathrm{R}^{2} / \nu\right)$, the density and magnetic field become constant along the lines of generated motion, and the fluxtube is "splitting" into four independent tubes. In the opposite case of a sequence of mutually incoherent (including the plane of polarization) short $\left(T<\tau_{\nu}\right.$ ) wave trains, a kind of stochastic motion is induced, which results in a diffusive broadening of a tube and ends up in a complete "dissolving" of fluxtube in the ambient plasma.

As it was mentioned the effects described above exist even in the absence of any mechanism of absorption. In the presence of absorption $z$-component of ponderomotive force directed along the magnetic field appear.

Other mechanism of generation of magnetosonic flows and in this case also the current drives is connected just with the absorption of the oscillation energy. Formally, the expression for $f$ has the same form as Eq. (23), but now the terms directly connected with the absorption are taken into account. Besides the absorption due to the usual dissipative processes for fluxtubes nondissipative mechanisms of absorption described above are quite efficient. For example, in the case of resonance damping with the damping rate (10) the energy of bending oscillations is pumping into the resonant layer where the dissipation occurs. Respectively, the whole momentum of oscillations is transferred to plasma in the narrow layer, causing the strong upward mass flow along the magnetic field. The corresponding force can be estimated as follows

$$
\mathrm{f}_{z} \sim \alpha \frac{\mathrm{B}^{2}}{4 \pi} \frac{|\tilde{\mathrm{v}}|^{2}}{\mathrm{a}^{2} \mathrm{R}}
$$

Now, if the absorption is provided mostly in electron (or ion) component of plasma the generation of currents inside the tube as well as outside occurs. As in the case of secondary flows, the absorption of momentum leads to the current drives along the fluxtubes and the absorption of angular momentum leads to the generation of azimuthal currents.

\section{THE ENSEMbles OF FLUXTUBES}

This part of paper deals with the effects accompanying the propagation of long-wave MHD-oscillations in a plasma containing small-scale inhomogeneities, namely, the ensembles of magnetic fluxtubes. In the first two sections we consider the ensemble of fluxtubes far removed from each other, while in the third section we discuss briefly the tightly settled fluxtubes. 
3.1. Resonance damping of sound waves in system of fluxtubes

The system of fluxtubes has an interesting feature: the longwave acoustic oscillations in the system are damped due to the effect similar to Landau damping which consists in the resonance excitation of bending waves propagating along the fluxtubes (Ryutov and Ryutova, 1976). The bending oscillations propagate along the fluxtube separate with the phase velocity defined by Eq. (1) and if the angle $\theta$ between the direction of sound propagation and fluxtube direction satisfies the condition

$$
s=c_{b} \cos v
$$

then the resonant transfer of sound wave energy to the energy of fluxtube oscillations takes place.

Since in general the density and the magnetic field inside the different fluxtubes are different, so the velocity changes from one fluxtube to another, and hence, at each propagation angle there are tubes for which the condition (27) is satisfied and which absorb the energy of sound waves.

We define the distribution function of fluxtubes $f(r, \eta)$ over their radius $R$ and parameter $\eta=\frac{\rho_{i}}{\rho_{e}}$ (we consider that $R$ is much less than the average distance between fluxtubes $L$ which is much less than wavelength: $\mathrm{R} \ll \mathrm{L} \ll \lambda)$ :

$$
\sigma=\int_{0}^{\infty} \int \mathrm{dRd} n \mathrm{f}(\mathrm{R}, \eta)
$$

where $\sigma$ is the total fraction of volume occupied with fluxtubes ( $\sigma \sim$ $\left.\sim \frac{\mathrm{R}^{2}}{\mathrm{~L}^{2}} \ll 1\right)$. The damping rate of sound oscillations has a form:

$$
\nu_{s}=\pi k s \sin ^{2} \theta\left\{\begin{array}{l}
g\left(n_{0}\right), n_{0}>0 \\
0, n_{0}<0
\end{array} ; n_{0}=\frac{2 \cos ^{2} \theta}{\gamma}-1\right.
$$

where we introduce the function

$$
g(n)=\int_{0}^{\infty} f(r, n) d R .
$$

If we make the natural assumption that $\rho_{i}$ changes with respect to $\rho_{e}$ not more than by several times from one tube to another, then $g\left(n_{0}\right) \sim \sigma$, and for damping rate we can write the following estimate:

$$
\nu_{\mathrm{s}} \sim \sigma \mathrm{ks}
$$

which holds only for those values of $\theta$, where $n_{0}>0$, i.e. where $\cos \theta>(\gamma / 2)^{\frac{1}{2}}$. For monatomic gas the corresponding region is quite narrow, $\theta \leqq 5^{\circ}$, so that it is essential to take into account the noncollinearity of separate fluxtubes. Let $h(\vec{n})$ be the distribution function of 
fluxtubes over the directions. $\vec{n}$ is the unit vector directed along the tube's axis. We can normalize $h(\vec{n})$ as follows:

$$
\sigma=\int h(\overrightarrow{\mathrm{n}}) \mathrm{d} \sigma \text {. }
$$

do is the element of solid angle. Then for isotropic distribution of fluxtubes the damping rate of sound oscillations has a form

$$
\frac{\nu_{s}}{\mathrm{ks}}=\frac{1}{4 \sqrt{2}} \frac{\sigma \gamma[2-\gamma(1+\eta)]}{\sqrt{\gamma(1+\eta)}} \text {. }
$$

So magnetic fluxtubes absorb the energy of outer motions at the photosphere level during the time of the order of $\nu_{s}{ }^{-1}$. Then the process of energy accumulation goes on, and in a time $\alpha_{\mathrm{rad}^{-1}}$ which is much greater $\quad\left(\alpha_{\mathrm{rad}}^{-1}>v_{s}{ }^{1}\right)$, fluxtubes release their energy due to radiation of secondary acoustic waves at the higher layers of atmosphere.

\subsection{Scattering of sound waves by fluxtubes}

The detailed theory of scattering of sound waves by magnetic fluxtubes (Ryutov and Ryutova, 1976) shows that there are resonances in scattering due to the existence of weakly damped natural oscillations of fluxtube. The main contribution to damping is of dipole mode, i.e. the bending oscillations. The damping rate of acoustic wave with frequency $\omega$ has a form

$$
\nu_{\text {scatt }}=\int \frac{f(R, \eta)}{\pi R^{2}} \beta(\eta, R, \omega) d \eta d \omega d R
$$

where $\beta$ is some function containing the resonance denominators

$$
\beta \sim \frac{\Omega^{2}}{(\omega-\Omega)^{2}+\alpha_{\text {rad }}^{2}} ; \Omega=k s \sqrt{\frac{2}{\gamma(1+n)}}
$$

For the narrow region of $=$ one can perform integration over $n$ and $\mathrm{R}$ resulting

$$
\nu_{\text {scatt }}=\pi s k \sin ^{2} \theta \cdot g\left(n_{0}\right)
$$

which formally coincides with (28). We imply that no $>0$; in the opposite case the scattering becomes nonresonant and damping rate becomes much smaller: $\nu_{\text {scatt }} \sim$ oks $(\mathrm{kR})^{2}$.

Note that if the condition $(k R)^{2}<\sigma$ is satisfied the scattering is negligible.

In the case of comparatively short waves $(R<\lambda<L$ L) the damping is completely determined by scattering. In this case the damping rate $\nu_{s}$ appears to be smaller than $\alpha_{\text {rad }}$ and this means that direct scattering of primary acoustic wave into the secondary sound waves without the preliminary accumulation of energy in natural oscillations of fluxtubes takes place. In other words for short waves one has $\nu_{S}=$ $=\nu_{\text {scatt }}$, where $\nu_{\text {scatt }}$ is defined by Eq. (29). 
Recently resonant scattering of sound waves by slender fluxtubes was studied by Bogdan (1989), who emphasized the importance of this effect for the diagnostics of the structure of magnetic flux concentrations across the solar surface. This may be possible because near the resonance the elastic scattering cross section (per unit length of the fluxtube) is comparable to the wavelength of the incident solar $p$ mode, which for slender fluxtubes may exceed both the geometrical and non-resonant scattering cross sections by several orders of magnitude.

\subsection{Enhanced dissipation by tightly packed fluxtubes}

Until now we have discussed the problems connected with isolated fluxtubes and ensembles of fluxtubes widely separated from each other. In the solar atmosphere the situation when fluxtubes are tightly packed seems quite realistic, so the question arises what the effects are that accompany the propagation of long-wave MHD oscillations in a plasma with random inhomogeneities, when the density, temperature, and magnetic field change by a large amount over a distance small as compared with the wavelength. Intuitively it seems that the largescale perturbations will propagate in quite the same way as in a homogeneous plasma, with the difference that the density, velocity, etc., will be replaced by some averaged quantities, and that intuitive conclusions should turn out to be more or less correct. It is however far from easy to find an explicit procedure for even the derivation of the averaged equations, especially for a real 3-D problem.

There are a few cases in which one can write the averaged equations in explicit form (Ryutova and Persson, 1984). It is interesting that such systems reveal some less obvious properties. One is that unlike the case of a homogeneous plasma, the wave propagation in a plasma with random (and strong) inhomogeneities is accompanied by a vortex motion of the plasma. These motions have the same scale as the fluxtube cross sections. Besides, because of the large gradients of velocity and temperature that are associated with tightly packed fluxtubes, enhanced dissipation of MHD waves can take place. The dissipation rates associated with both the viscosity and thermal conductivity are $(\lambda / R)^{2}$ (with $\lambda \gg R$ ) times larger as compared with the homogeneous case. Ohmic dissipation gives only a minor contribution to this effect. Thus although dissipative effects are believed to be small in the solar atmosphere, these effects may strongly influence the dynamics of inhomogeneous regions.

\section{REFERENCES}

1. Stenflo, J.0. (1989) The Astronomy and Astrophysics Review 1, 3.

2. Priest, E.R. (1982) Solar Magnetohydrodynamics, Dodrecht, D.Reidel Pub1. Co.

3. Ryutov, D.D. \& Ryutova, M.P. (1976) Sov. Phys. JETP, 43, 491.

4. Defouw, R.J. (1976), Ap.J. 209, 266.

5. Ryutova, M.P. (1977) Proc. of XIII Int. Conf. on Ionized Gases,p. 859.

6. Ionson, J.A. (1978) Ap.J. 226, 650 .

7. Spruit, H.C. (1981a) Astr. Ap., 98, 155. 
8. Roberts, B. \& Webb, A.R. (1978) Solar Phys. 59, 249.

9. Webb, A.R. \& Roberts, B. (1979) Solar Phys. 64, 77.

10. Rae, I.C. \& Roberts, B. (1982) Ap. J. 256, $7 \overrightarrow{61}$.

11. Hollweg, J.V. (1982) Ap. J. 257, 345.

12. Ryutova, M.P. (1989) Proc. of AGU Chapman Conf. on Physics of Magnetic Flux Ropes (in press).

13. Ryutova, M.P.(1988), JETP, 94, 138.

14. Roberts, B. \& Mangeney, A. (1982) Mon. Not. Roy. Astr. Soc. 198,7p.

15. Roberts, B. (1985) Phys. Fluids, $28,3280$.

16. Molotovshchikov, A.L. \& Ruderman, M.S. (1987) Solar Phys.

17. Ryutova, M.P. (1986) Proc. of Joint Varenna-Abastumani Int. Schoo1 on Plasma Astrophys., ESA, p.71.

18. Ryutov, D.D. \& Ryutova, M.P. (1989) Proc. of AGU Chapman Conf. on Physics of Magnetic Flux Ropes (in press).

19. Bogdan, T. (1989) Ap. J. (in press).

20. Bogdan, T \& Zweibe1, E.G. (1985) Ap. J. 298, 867.

21. Bogdan, T. \& Zweibe1, E.G. (1987) Ap. J.318, 888 .

22. Bogdan, T. \& Cattaneo, F. (1989) Ap. J. (in press).

23. Ryutova, M. \& Persson, M. (1984) Physica Scripta, 29, 353.

24. Weiss, N.O. (1981) J.Fluid Mech., 108, 247; 273.

25. Hasan, S.S. \& Schüssler, M. (1985) Astr. Ap. 151, 69.

26. Ferriz-Mas, A. (1988) Phys. Fluids, 31, 2583.

27. Hollweg, J. (1987) Ap. J. 317, 918.

28. Hollweg, J., Yang, G., Cadez, V.M. \& Gakovic (1989) Ap.J. (in press).

29. Seehafer, N. (1988) Proc. Int. Workshop on Reconnection in Space Plasma, Potsdam, p. 5.

30. Henoux, J.C. \& Somov, B.V. (1987) Astr. Ap., 185, 306.

31. Spruit, H.C. (1981) in The Sun as a Star, ed. S.Jordan (Washington, NASA SP-450) p. 385.

32. Thomas, J.H. (1985) Proc. Solar Optical Telescope Workshop, "Theoretical Problems in Solar Physics", Munich.

33. Priest, E.R. (1988) Proc. of Joint Varenna-Abastumani Int. School on Plasma Astrophysics, ESA, p. 189.

34. Spruit, H.C. (1981b) Astr. Ap. 102, 129.

35. Roberts, B. \& Webb, A.R. (1978) Solar Phys. 56, 5.

36. Spruit, H.C. \& Zweibel, E.G. (1979) Solar Phys. 62, 15.

37. Spruit, H.C. (1979) Solar Phys. 61, 363.

38. Timofeev, A.V. (1970) Usp. Fiz. Nauk, 102, 185.

39. Hollweg, J. (1979) Solar Phys. 62, 227.

40. Heyaeverts, J. \& Priest, E. (19 $\overline{83})$ Astr. Ap. 117, 220.

41. Sudan, R. \& Similon, P. (1988) Proc. of Joint Varenna-Abastumani Int. School on Plasma Astrophysics, ESA, p. 63.

42. Sakurai, T. \& Granik, A. (1984) Ap. J. 177, 404.

43. Ryutova, M.P. (1981) Sov. Phys. JETP, 53(3), 529.

44. Kadomtsev, B.B., Mikhailovsky, A.B. \& Timofeev, A.V. (1964) JETP, 47, 2267.

45. Ostrovsky, L.A., Rybak, S.A. \& Tsimring, L. (1986) Usp. Fiz. Nauk, $150,417$.

46. Dikasov, V.M., Rudakov, L.I. \& Ryutov, D.D. (1965) JETP, 48, 913.

47. Coppi, B., Rosenbluth, M.N. \& Sudan, R. (1969) Ann. Phys. $\underline{55}, 207$.

48. Leibovich, S. (1970) J. Fluid Mech. 51, 625. 